\title{
Announcement of population data Y-chromosome STR haplotypes in East Timor: Forensic evaluation and population data
}

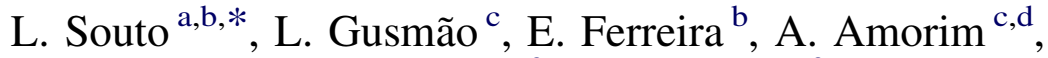 \\ F. Côrte-Real ${ }^{\mathrm{e}, \mathrm{f}}$, D.N. Vieira ${ }^{\mathrm{e}, \mathrm{f}}$ \\ ${ }^{a}$ Centro de Biologia Celular, Universidade de Aveiro, Portugal \\ ${ }^{\mathrm{b}}$ Departamento de Biologia, Universidade de Aveiro, Portugal \\ ${ }^{\mathrm{c}}$ Instituto de Patologia e Imunologia Molecular da Universidade do Porto (IPATIMUP), Portugal \\ ${ }^{\mathrm{d}}$ Faculdade de Ciências, Universidade do Porto, Portugal \\ ${ }^{\mathrm{e}}$ Instituto Nacional de Medicina Legal, Delegação de Coimbra, Portugal \\ ${ }^{\mathrm{f}}$ Faculdade de Medicina, Universidade de Coimbra, Portugal
}

Received 7 December 2004; received in revised form 25 February 2005; accepted 26 February 2005

Available online 2 April 2005

Population: One hundred and thirty eight unrelated individuals from several districts of East Timor, including the Oecussi-Ambeno enclave and island of Ataúro (Fig. 1).

DNA extraction: The DNA was extracted by the Chelex procedure according to Walsh et al. [1] over a $1.2 \mathrm{~mm} \mathrm{FTA}^{\circledR}$ card punch.

PCR: Amplifications were performed using the PowerPlex ${ }^{\circledR}$ Y System kit (Promega Corporation), following the manufacturers' instructions.

Typing: Typing was performed in an ABI 310 automatic sequencer (AB, Applied Biosystems) with the Genescan 2.1 Analysis software. Allele designation was based on comparison with the allelic ladders provided in the PowerPlex ${ }^{\circledR} \mathrm{Y}$ System kit (Promega Corporation).

Results: See Table 1 and Fig. 2.

Quality control: Proficiency testing of the GEP-ISFG Working Group (http://www.gep-isfg.org).

Analysis of data: Haplotype frequencies were estimated by gene counting. Haplotype diversities were calculated according to Nei [2] using the ARLEQUIN software, version 2.000 [3]. Analysis of molecular variance (AMOVA) results were summarised in the form of $R_{\mathrm{st}}$ values and assessed for statistical significance using a Monte-Carlo test as implemented in the Arlequin software. In population comparisons

\footnotetext{
* Corresponding author. Tel.: +351 234370783; fax: +351234426408 .

E-mail address: 1souto@bio.ua.pt (L. Souto).
}

DYS385 was not considered and the number of repeats in DYS389I was subtracted from DYS389II. The $R_{\mathrm{st}}$ values among populations were used to draw a neighbour-joining tree with the neighbour routine of the PHYLIP 3.5c software package [4] and the tree was further visualized with the Treeview software [5].

Access to the data: http://www.bio.ua.pt/POPGeneTIMOR/popgene_timor.htm.

Other remarks: Most loci display high diversity values varying between 0.5241 and 0.9604 . A list of the haplotypes found in our population sample is depicted in Table 1. The present haplotype data have been already deposited in the YHRD (Y-STR Haplotype Reference Database). In this sample, we found 113 different haplotypes, 101 unique, 2 with two records (H37 and H105), 7 with three records (H4, $\mathrm{H} 6, \mathrm{H} 16, \mathrm{H} 28, \mathrm{H} 29, \mathrm{H} 43$ and $\mathrm{H} 63$ ), and 3 representing the most frequent haplotypes with 4 records (H2, H12 and H59).

Considering the whole set of Y-STRs included in the PowerPlex ${ }^{\circledR}$ Y System kit, the haplotype diversity was $0.9957 \pm 0.0017$. For the "minimal haplotype", the observed diversity value was $0.9939( \pm 0.0020)$, which is lower than the value (0.9996) reported by Lessig et al. [6] for the Asian samples in the YHRD. This difference is not unexpected since the whole Asian database results from samples of many different Asian populations.

East Timor (República Democrática de Timor Leste), a former Portuguese colony and now the newest independent country, is situated in the east part of Timor island, the 


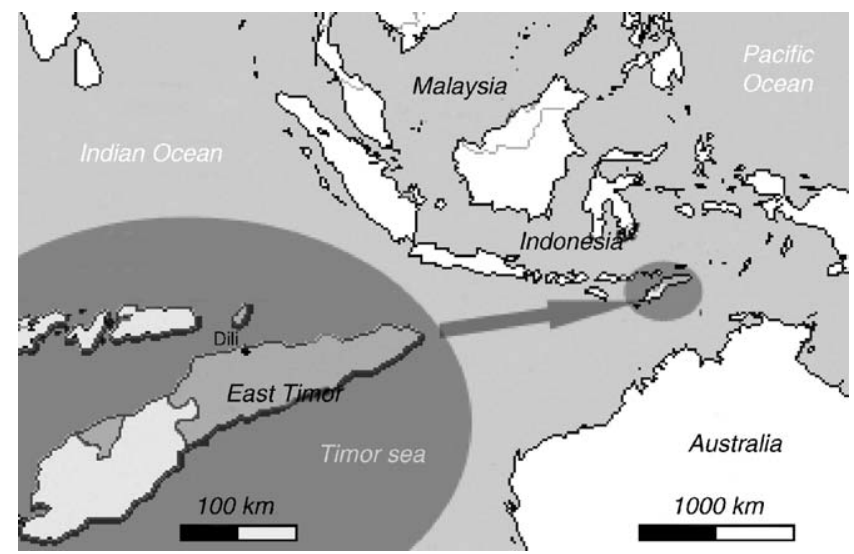

Fig. 1. Geographical localization of East Timor.

largest of Lesser Sunda Islands, South-eastern Asia, northwest of Australia (Fig. 1).

Human occupation in East Timor has been recently dated from before 30,000 BP [7]. The present East Timor population reflects centuries of successive immigration of people including mainly Melanesian, Malayo-Polynesian but also, more recently, some Chinese, Arabic, Indian and African, besides the European, especially Portuguese [8]. The result is an ethnolinguistic mosaic for which different numbers of groups and classifications have been reported. Portuguese anthropological missions in 1950s and 1960s have detected 31 groups [9].

As a result of an international cooperation with East Timor Health Ministry and Universidade Nacional de Timor Leste, with several Portuguese institutions, we developed a project, POP-Gene Timor, to characterize the genetic variation in the population of East Timor [10]. This project offers the first haplotype frequency study available to local law authorities, while giving some clues on the possible origins of present East Timor population, as regarded by Y chromosome haplotype analysis and comparisons with populations from the region of concern.

Minimal haplotype data from our East Timor sample were compared with data available in the YHRD for neighbour populations and also with a sample from Portugal. The following populations were used: Djakarta, Indonesia $(n=41)$ [11], Trobriand Islands, Highland and Coastal Papua New Guinea ( $n=58, n=29$ and $n=25$, respectively) [11], Luzon, Philippines $(n=211)$ [12], Macao, China $(n=57)$ [13], Chengdu, China $(n=55) \quad$ [14], Taiwan $(n=466)$ [15] and Portugal $(n=212)$ [16].

Fig. 2 shows a neighbour joining tree based on pairwise $\Phi_{\text {st }}$ distances between all the samples except the Portuguese.

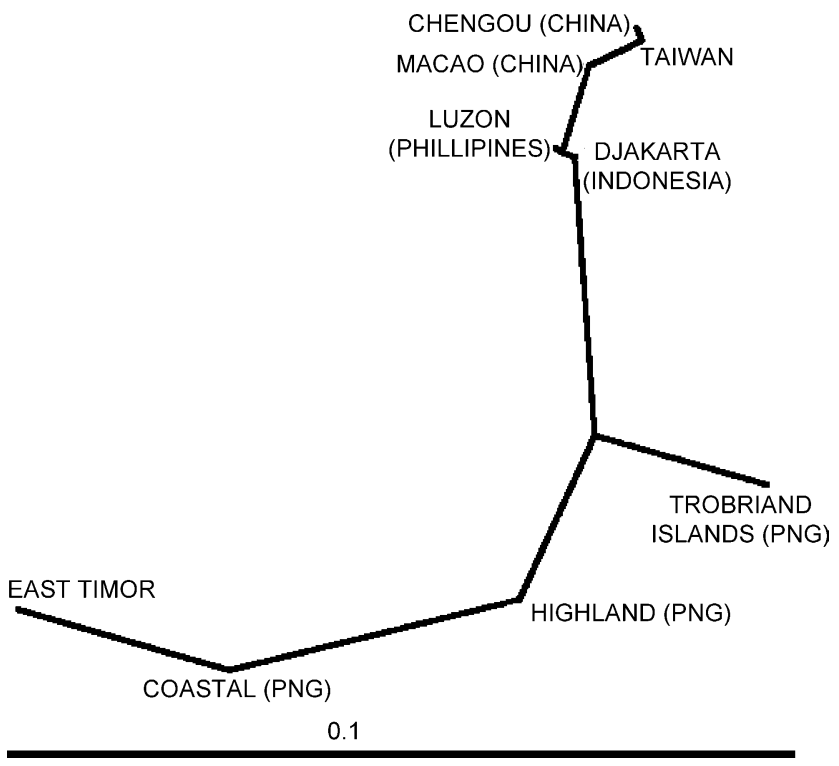

Fig. 2. Unrooted NJ tree based on $\Phi_{\text {st }}$ distances. PNG: Papua New Guinea. 
Table 1

Y-chromosome haplotype distribution in 138 individuals from East Timor (Timor Leste) population

\begin{tabular}{|c|c|c|c|c|c|c|c|c|c|c|c|c|}
\hline & $N$ & DYS19 & Y389I & Y389II & Y390 & Y391 & Y392 & Y393 & Y385 & Y437 & Y438 & Y439 \\
\hline H1 & 1 & 15 & 12 & 28 & 20 & 10 & 12 & 13 & $14-20$ & 14 & 10 & 11 \\
\hline $\mathrm{H} 2$ & 4 & 14 & 13 & 31 & 25 & 10 & 13 & 12 & $12-14$ & 14 & 9 & 11 \\
\hline H3 & 1 & 15 & 14 & 32 & 22 & 10 & 11 & 13 & $13-13$ & 14 & 10 & 10 \\
\hline $\mathrm{H} 4$ & 3 & 16 & 12 & 30 & 21 & 10 & 12 & 13 & $14-17$ & 14 & 10 & 12 \\
\hline H5 & 1 & 14 & 14 & 30 & 25 & 11 & 11 & 13 & $12-16$ & 14 & 10 & 13 \\
\hline H6 & 3 & 16 & 12 & 29 & 23 & 10 & 14 & 13 & $11-14$ & 14 & 10 & 11 \\
\hline $\mathrm{H} 7$ & 1 & 14 & 15 & 30 & 23 & 10 & 14 & 14 & $13-19$ & 14 & 9 & 13 \\
\hline $\mathrm{H} 8$ & 1 & 15 & 13 & 30 & 21 & 10 & 11 & 14 & $14-14$ & 14 & 11 & 11 \\
\hline H9 & 1 & 14 & 12 & 29 & 24 & 10 & 14 & 13 & $12-13$ & 14 & 9 & 13 \\
\hline $\mathrm{H} 10$ & 1 & 16 & 13 & 29 & 23 & 11 & 13 & 13 & $13-15$ & 15 & 10 & 13 \\
\hline H11 & 1 & 15 & 13 & 29 & 21 & 10 & 12 & 13 & $14-18$ & 14 & 10 & 11 \\
\hline H12 & 4 & 13 & 13 & 30 & 22 & 11 & 11 & 13 & $15-17$ & 14 & 10 & 13 \\
\hline H13 & 1 & 15 & 14 & 32 & 23 & 10 & 11 & 13 & $13-13$ & 14 & 10 & 10 \\
\hline H14 & 1 & 14 & 13 & 30 & 24 & 10 & 15 & 14 & $12-12$ & 14 & 9 & 12 \\
\hline H15 & 1 & 14 & 12 & 28 & 24 & 10 & 13 & 14 & $12-13$ & 14 & 8 & 12 \\
\hline H16 & 3 & 16 & 14 & 31 & 22 & 10 & 13 & 12 & $13-13$ & 14 & 10 & 12 \\
\hline H17 & 1 & 15 & 12 & 28 & 21 & 10 & 12 & 13 & $11-20$ & 14 & 10 & 11 \\
\hline H18 & 1 & 16 & 13 & 31 & 23 & 10 & 14 & 13 & $12-13$ & 14 & 9 & 13 \\
\hline H19 & 1 & 15 & 12 & 28 & 21 & 10 & 12 & 13 & $16-19$ & 14 & 10 & 12 \\
\hline $\mathrm{H} 20$ & 1 & 15 & 12 & 28 & 23 & 10 & 14 & 13 & $14-14$ & 14 & 10 & 12 \\
\hline $\mathrm{H} 21$ & 1 & 14 & 14 & 31 & 21 & 10 & 11 & 13 & $12-14$ & 14 & 10 & 11 \\
\hline $\mathrm{H} 22$ & 1 & 15 & 14 & 30 & 23 & 10 & 13 & 13 & $11-15$ & 14 & 10 & 11 \\
\hline $\mathrm{H} 23$ & 1 & 15 & 12 & 29 & 21 & 10 & 12 & 14 & $14-19$ & 14 & 10 & 12 \\
\hline $\mathrm{H} 24$ & 1 & 15 & 12 & 29 & 24 & 10 & 13 & 13 & $11-14$ & 15 & 12 & 12 \\
\hline $\mathrm{H} 25$ & 1 & 15 & 12 & 28 & 22 & 10 & 12 & 13 & $14-19$ & 14 & 10 & 11 \\
\hline H26 & 1 & 14 & 13 & 28 & 23 & 10 & 14 & 14 & $13-21$ & 14 & 10 & 12 \\
\hline $\mathrm{H} 27$ & 1 & 15 & 13 & 29 & 25 & 11 & 13 & 13 & $12-15$ & 16 & 11 & 12 \\
\hline $\mathrm{H} 28$ & 3 & 14 & 14 & 29 & 23 & 10 & 14 & 14 & $13-20$ & 14 & 10 & 12 \\
\hline $\mathrm{H} 29$ & 3 & 15 & 13 & 29 & 22 & 10 & 11 & 14 & $14-19$ & 14 & 11 & 11 \\
\hline $\mathrm{H} 30$ & 1 & 16 & 13 & 29 & 23 & 11 & 13 & 12 & $13-15$ & 15 & 10 & 13 \\
\hline H31 & 1 & 15 & 12 & 28 & 21 & 10 & 12 & 13 & $13-20$ & 14 & 10 & 11 \\
\hline H32 & 1 & 16 & 12 & 28 & 20 & 10 & 12 & 13 & $16-19$ & 14 & 10 & 12 \\
\hline H33 & 1 & 15 & 13 & 29 & 23 & 10 & 13 & 13 & $12-14$ & 15 & 12 & 11 \\
\hline H34 & 1 & 16 & 13 & 30 & 22 & 10 & 11 & 13 & $14-17$ & 14 & 11 & 11 \\
\hline H35 & 1 & 15 & 12 & 28 & 23 & 10 & 14 & 13 & $13-14$ & 14 & 10 & 11 \\
\hline H36 & 1 & 15 & 14 & 31 & 21 & 10 & 11 & 13 & $13-16$ & 14 & 11 & 13 \\
\hline H37 & 2 & 15 & 13 & 29 & 22 & 10 & 11 & 14 & $14-18$ & 14 & 11 & 11 \\
\hline H38 & 1 & 15 & 12 & 28 & 24 & 10 & 13 & 13 & $12-16$ & 15 & 10 & 12 \\
\hline H39 & 1 & 13 & 13 & 31 & 23 & 10 & 11 & 12 & $15-16$ & 14 & 10 & 12 \\
\hline $\mathrm{H} 40$ & 1 & 16 & 13 & 30 & 23 & 11 & 14 & 12 & $13-15$ & 16 & 10 & 12 \\
\hline H41 & 1 & 15 & 12 & 28 & 20 & 10 & 12 & 13 & $14-20$ & 14 & 10 & 13 \\
\hline $\mathrm{H} 42$ & 1 & 15 & 13 & 29 & 21 & 11 & 10 & 13 & $14-14$ & 14 & 10 & 11 \\
\hline H43 & 3 & 14 & 12 & 29 & 24 & 10 & 14 & 13 & $12-12$ & 14 & 9 & 13 \\
\hline H44 & 1 & 15 & 12 & 28 & 22 & 10 & 12 & 13 & $14-20$ & 14 & 10 & 11 \\
\hline H45 & 1 & 14 & 12 & 27 & 25 & 11 & 14 & 14 & $14-15$ & 16 & 11 & 13 \\
\hline H46 & 1 & 15 & 12 & 28 & 22 & 10 & 12 & 13 & $13-21$ & 14 & 10 & 11 \\
\hline $\mathrm{H} 47$ & 1 & 16 & 12 & 28 & 21 & 10 & 12 & 13 & $14-15$ & 16 & 10 & 12 \\
\hline H48 & 1 & 15 & 12 & 29 & 21 & 10 & 13 & 13 & $15-16$ & 14 & 10 & 11 \\
\hline H49 & 1 & 15 & 12 & 29 & 24 & 11 & 13 & 13 & $12-15$ & 16 & 11 & 11 \\
\hline H50 & 1 & 14 & 15 & 31 & 23 & 10 & 12 & 13 & $12-19$ & 15 & 9 & 11 \\
\hline H51 & 1 & 16 & 14 & 31 & 22 & 11 & 13 & 12 & $13-13$ & 14 & 10 & 13 \\
\hline H52 & 1 & 17 & 12 & 28 & 25 & 10 & 13 & 12 & $12-19$ & 14 & 10 & 11 \\
\hline H53 & 1 & 16 & 15 & 31 & 22 & 10 & 11 & 14 & $14-19$ & 14 & 11 & 12 \\
\hline H54 & 1 & 14 & 12 & 28 & 21 & 10 & 12 & 13 & $14-14$ & 14 & 10 & 11 \\
\hline H55 & 1 & 14 & 13 & 30 & 22 & 10 & 13 & 13 & $13-22$ & 15 & 9 & 12 \\
\hline H56 & 1 & 16 & 12 & 29 & 21 & 10 & 12 & 13 & $14-17$ & 14 & 10 & 12 \\
\hline H57 & 1 & 13 & 13 & 30 & 22 & 10 & 11 & 13 & $17-17$ & 14 & 10 & 12 \\
\hline
\end{tabular}


Table 1 (Continued)

\begin{tabular}{|c|c|c|c|c|c|c|c|c|c|c|c|c|}
\hline & $N$ & DYS19 & Y389I & Y389II & Y390 & Y391 & Y392 & Y393 & Y385 & Y437 & Y438 & Y439 \\
\hline H58 & 1 & 16 & 13 & 30 & 23 & 10 & 15 & 14 & $12-14$ & 12 & 10 & 12 \\
\hline H59 & 4 & 14 & 12 & 28 & 24 & 10 & 14 & 14 & $12-19$ & 14 & 10 & 11 \\
\hline H60 & 1 & 15 & 12 & 28 & 21 & 10 & 12 & 13 & $14-20$ & 14 & 10 & 11 \\
\hline H61 & 1 & 15 & 13 & 31 & 24 & 10 & 13 & 14 & $16-19$ & 14 & 10 & 12 \\
\hline H62 & 1 & 14 & 12 & 29 & 24 & 10 & 14 & 13 & $11-12$ & 14 & 9 & 12 \\
\hline H63 & 3 & 15 & 12 & 28 & 20 & 10 & 12 & 13 & $14-19$ & 14 & 10 & 11 \\
\hline H64 & 1 & 16 & 13 & 29 & 23 & 11 & 14 & 13 & $13-15$ & 16 & 10 & 12 \\
\hline H65 & 1 & 14 & 12 & 28 & 23 & 10 & 14 & 12 & $15-18$ & 15 & 11 & 12 \\
\hline H66 & 1 & 15 & 12 & 28 & 24 & 10 & 13 & 13 & $12-12$ & 15 & 10 & 13 \\
\hline H67 & 1 & 15 & 13 & 30 & 21 & 11 & 11 & 13 & $14-18$ & 14 & 11 & 11 \\
\hline H68 & 1 & 14 & 14 & 29 & 23 & 10 & 14 & 13 & $13-20$ & 14 & 10 & 12 \\
\hline H69 & 1 & 16 & 12 & 28 & 20 & 10 & 12 & 13 & $17-18$ & 14 & 10 & 11 \\
\hline H70 & 1 & 15 & 12 & 29 & 21 & 9 & 12 & 13 & $12-14$ & 14 & 10 & 12 \\
\hline H71 & 1 & 15 & 12 & 29 & 24 & 10 & 13 & 13 & $12-12$ & 15 & 10 & 13 \\
\hline $\mathrm{H} 72$ & 1 & 16 & 12 & 28 & 21 & 10 & 12 & 13 & $14-19$ & 14 & 10 & 11 \\
\hline H73 & 1 & 14 & 14 & 29 & 23 & 10 & 14 & 14 & $13-19$ & 14 & 10 & 11 \\
\hline H74 & 1 & 15 & 12 & 28 & 20 & 10 & 12 & 13 & $14-19$ & 14 & 10 & 12 \\
\hline H75 & 1 & 15 & 12 & 28 & 22 & 10 & 12 & 13 & $14-14$ & 14 & 10 & 11 \\
\hline H76 & 1 & 15 & 13 & 28 & 22 & 10 & 14 & 12 & $11-11$ & 15 & 10 & 10 \\
\hline H77 & 1 & 16 & 13 & 31 & 21 & 11 & 11 & 13 & $14-17$ & 14 & 11 & 11 \\
\hline H78 & 1 & 16 & 14 & 31 & 22 & 9 & 13 & 12 & $13-13$ & 14 & 10 & 12 \\
\hline H79 & 1 & 15 & 12 & 28 & 24 & 10 & 14 & 14 & $12-19$ & 14 & 10 & 11 \\
\hline H80 & 1 & 15 & 13 & 29 & 25 & 11 & 13 & 13 & $12-12$ & 16 & 11 & 12 \\
\hline H81 & 1 & 14 & 12 & 28 & 25 & 11 & 13 & 14 & $14-15$ & 16 & 11 & 11 \\
\hline H82 & 1 & 13 & 13 & 30 & 24 & 10 & 11 & 14 & $14-18$ & 14 & 10 & 12 \\
\hline H83 & 1 & 14 & 13 & 30 & 24 & 10 & 11 & 12 & $12-15$ & 16 & 10 & 11 \\
\hline H84 & 1 & 15 & 13 & 31 & 25 & 11 & 14 & 13 & $12-12$ & 14 & 9 & 12 \\
\hline H85 & 1 & 14 & 14 & 29 & 23 & 10 & 14 & 14 & $13-20$ & 14 & 10 & 13 \\
\hline H86 & 1 & 14 & 12 & 28 & 24 & 10 & 14 & 14 & $12-19$ & 14 & 10 & 12 \\
\hline H87 & 1 & 16 & 12 & 29 & 21 & 10 & 12 & 13 & $14-19$ & 14 & 10 & 11 \\
\hline H88 & 1 & 16 & 13 & 31 & 21 & 10 & 11 & 15 & $16-17$ & 14 & 9 & 12 \\
\hline H89 & 1 & 15 & 14 & 32 & 22 & 10 & 11 & 13 & $13-13$ & 14 & 10 & 11 \\
\hline H90 & 1 & 14 & 13 & 28 & 23 & 10 & 14 & 14 & $13-19$ & 14 & 10 & 11 \\
\hline H91 & 1 & 14 & 13 & 31 & 24 & 10 & 11 & 14 & $14-16$ & 14 & 10 & 13 \\
\hline H92 & 1 & 15 & 13 & 29 & 22 & 10 & 11 & 15 & $14-19$ & 14 & 11 & 12 \\
\hline H93 & 1 & 14 & 13 & 31 & 24 & 11 & 15 & 14 & $12-12$ & 14 & 9 & 11 \\
\hline H94 & 1 & 16 & 12 & 30 & 21 & 10 & 12 & 13 & $14-17$ & 14 & 10 & 13 \\
\hline H95 & 1 & 15 & 14 & 32 & 22 & 10 & 11 & 13 & $13-14$ & 14 & 10 & 10 \\
\hline H96 & 1 & 16 & 13 & 29 & 23 & 11 & 14 & 12 & $13-15$ & 16 & 10 & 12 \\
\hline H97 & 1 & 15 & 13 & 29 & 21 & 10 & 11 & 14 & $13-18$ & 14 & 12 & 13 \\
\hline H98 & 1 & 15 & 14 & 31 & 20 & 10 & 11 & 13 & $13-16$ & 14 & 11 & 13 \\
\hline H99 & 1 & 16 & 13 & 31 & 22 & 10 & 11 & 13 & $12-13$ & 14 & 10 & 10 \\
\hline H100 & 1 & 14 & 13 & 29 & 23 & 10 & 13 & 13 & $12-18$ & 15 & 9 & 12 \\
\hline H101 & 1 & 15 & 13 & 29 & 24 & 11 & 13 & 13 & $11-13$ & 14 & 12 & 12 \\
\hline H102 & 1 & 13 & 14 & 31 & 22 & 11 & 11 & 13 & $14-16$ & 14 & 10 & 12 \\
\hline H103 & 1 & 15 & 13 & 29 & 25 & 10 & 13 & 13 & $12-15$ & 16 & 11 & 12 \\
\hline H104 & 1 & 17 & 13 & 29 & 23 & 11 & 14 & 12 & $13-15$ & 16 & 10 & 12 \\
\hline H105 & 2 & 15 & 13 & 30 & 22 & 10 & 11 & 14 & $14-18$ & 14 & 11 & 11 \\
\hline H106 & 1 & 15 & 13 & 29 & 25 & 11 & 13 & 13 & $12-15$ & 16 & 11 & 13 \\
\hline H107 & 1 & 14 & 14 & 30 & 26 & 11 & 11 & 13 & $12-16$ & 14 & 10 & 13 \\
\hline H108 & 1 & 14 & 14 & 29 & 23 & 10 & 14 & 14 & $13-18$ & 14 & 10 & 11 \\
\hline H109 & 1 & 14 & 13 & 29 & 23 & 10 & 13 & 13 & $12-20$ & 14 & 9 & 11 \\
\hline H110 & 1 & 15 & 14 & 29 & 23 & 10 & 14 & 14 & $13-21$ & 14 & 11 & 12 \\
\hline H111 & 1 & 15 & 12 & 28 & 24 & 10 & 13 & 13 & $12-12$ & 15 & 10 & 14 \\
\hline H112 & 1 & 15 & 12 & 28 & 21 & 10 & 12 & 13 & $14-14$ & 14 & 10 & 11 \\
\hline H113 & 1 & 15 & 12 & 28 & 24 & 10 & 13 & 13 & $12-13$ & 15 & 10 & 13 \\
\hline
\end{tabular}


Despite the Portuguese presence in Timor since the XVIth century, the genetic distance between these two populations is very high $\left(\Phi_{\mathrm{st}}=0.15099\right)$. When comparing East Timor with the remaining populations, significant $\Phi_{\text {st }}$ values $(P<0.05)$ were always obtained except for Coastal Papua New Guinea $\left(\Phi_{\mathrm{st}}=-0.00264 ; P=0.54054 \pm 0.0489\right)$.

Analysis of molecular variance (AMOVA) was performed for Timor and neighbouring populations (therefore, excluding Portugal). When all populations were considered as a single group, $6.67 \%$ of the genetic variation was found among populations. When two groups were defined (grouping Timor and Papua New Guinea samples in one group and the remaining populations in another) the variation among groups was $8.13 \%$ and among population within groups was $2.51 \%$, showing a significant genetic heterogeneity at both levels. When considering the Trobriand Islands in a third group, the percentage of variation between groups increased to 10.11 and between populations within the same group decreased to $1.26 \%$.

This paper follows the guidelines for publication of population data requested by the journal [17].

\section{Acknowledgment}

The authors would like to thank: Fundação Oriente; the East Timor Misnistry of Health, Universidade Nacional de Timor Leste; the Health Services of the former Portuguese

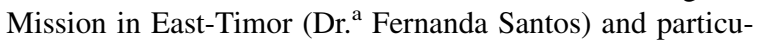
larly to the East Timorese volunteers. This work was partially supported by Fundação para a Ciência e a Tecnologia, Programa Operacional Ciência, Tecnologia e Inovação (POCTI).

\section{References}

[1] P.S. Walsh, D.A. Metzer, R. Higuchi, Chelex-100 as a medium for simple extraction of DNA for PCR-based typing from forensic material, BioTechniques 10 (1991) 506-513.

[2] M. Nei, Molecular Evolutionary Genetics, Columbia University Press, New York, 1987.

[3] S. Schneider, D. Roessli, L. Excoffier, Arlequin version 2.000. A Software for Population Genetics Data Analysis, University of Geneva, 2000.

[4] J. Felsenstein, PHYLIP: Phylogeny Inference Package Version 3.5c. Distributed by the Author, Department of Genetics, University of Washington, Seattle, 1993.
[5] R.D.M. Page, Treeview: an application to display phylogenetic trees on personal computers, Comput. Appl. Biosci. 12 (1996) 357-358.

[6] R. Lessig, S. Willuweit, M. Krawczak, F.-C. Wu, C.-E. Pu, W. Kim, L. Henke, J. Henke, J. Miranda, M. Hidding, M. Benecke, C. Schmitt, M. Magno, G. Calacal, F.C. Delfin, M.C.A. de Ungria, S. Elias, C. Augustin, Z. Tun, K. Honda, M. Kayser, L. Gusmão, A. Amorim, C. Alves, Y. Hou, C. Keyser, B. Ludes, M. Klintschar, U. Immel, B. Reichenpfader, B. Zaharova, L. Roewer, Asian online Y-STR Haplotype Reference Database, Legal Med. 5 (2003) S160-S163.

[7] S. O'Connor, M. Spriggs, P. Veth, Excavation at Lene Hara Cave establishes occupation in East Timor at least 30,00035,000 years ago, Antiquity 76 (2002) 45-50.

[8] A. Guedes, Thinking East Timor, Indonesia and Southeast Asia, Lusotopie (2001) 315-325.

[9] M.C. Almeida, Estudo Serológico dos grupos etnolinguísticos de Timor-Díli (sistema ABO), Instituto de Investigação Científica Tropical, Lisboa Estudos Ensaios e Documentos (1982) 141.

[10] L. Souto, L. Gusmão, A. Amorim, E. Ferreira, F. Corte-Real, D.N. Vieira, E.F. Cruz e Silva, POP-Gene TIMOR: first forensic DNA marker study of East-Timor people, International Congress Series, Progress in Forensic Genetics 1261 (2004) 201-203.

[11] M. Kayser, M. Krawczak, L. Excoffier, P. Dieltjes, D. Corach, V. Pascali, C. Gehrig, L.F. Bernini, J. Jespersen, E. Bakker, L. Roewer, P. de Knijff, An extensive analysis of Y-chromosomal microsatellite haplotypes in globally dispersed human populations, Am. J. Hum. Genet. 68 (2001) 990-1018.

[12] M. Tan, F. Delfin, G. Calacal, L. Roewer, M.C.A. De Ungria, Y-chromosome STR allele frequency distribution among male Filipinos in the National Capital Region for forensic applications, Philipp. J. Sci. 132 (2004) 95-101.

[13] L. Gusmão, A. Amorim, N. Martins, M.J. Prata, A. GonzalezNeira, M.V. Lareu, A. Carracedo, Y chromosome STR Haplotypes in Macau (SE China), Progress in Forensic Genetics, vol. 8, Elsevier Science, Amsterdam, 2000, pp. 324-326.

[14] M. Hidding, C. Schmitt, Haplotype frequencies and population data of nine Y-chromosomal STR polymorphisms in a German and a Chinese population, Forensic Sci. Int. 113 (2000) $47-53$.

[15] F.C. Wu, C.E. Pu, Multiplex DNA typing of short tandem repeat loci on Y chromosome of Chinese population in Taiwan, Forensic Sci. Int. 120 (2001) 213-222.

[16] L. Gusmão, C. Alves, S. Beleza, A. Amorim, Forensic evaluation and population data on the new Y-STRs DYS434, DYS437, DYS438, DYS439 and GATA A10, Int. J. Legal Med. 116 (2002) 139-147.

[17] P. Lincoln, A. Carracedo, Publication of population data of human polymorphisms, Forensic Sci. Int. 110 (2000) 3-5. 